\title{
Medizinische Texte im Wandel der Zeiten. Schriftträger und Buchtypen im nachchristlichen Ägypten*
}

\author{
Andrea Jördens
}

Nicht zuletzt durch die Arbeiten von Isabella Andorlini haben die Forschungen zu den antiken medizinischen Texten in den vergangenen Jahrzehnten bedeutenden Aufschwung genommen. Noch mehr als auf anderen Gebieten der griechischen Papyrologie galt es dabei stets als die vornehmste Aufgabe des Papyrologen, bislang unbekannte Quellen aufzufinden und sie der Wissenschaft zu erschließen. Wenn bei dem Durchgang durch die Sammlungen nichts dergleichen zu entdecken war, ließen sich vielleicht - so die zweitbeste Variante - neue Textzeugen zu schon anderweitig überlieferten Abhandlungen der großen Ärzte identifizieren. Sofern man weder Traktate von Vertretern der klassischen Medizin noch solche späterer Autoren fand, mochte man immerhin noch auf umfangreichere Rezeptsammlungen hoffen; wenn selbst das nicht, mußte man sich notfalls auch mit der Edition eines einzelnen Rezeptes begnügen. Im Vordergrund standen dabei durchweg sprachliche, aber mehr noch inhaltliche Fragen, da dies im wesentlichen, wenn nicht gar ausschließlich eine Domäne der Philologen oder wenigstens von Wissenschaftshistorikern mit philologischen Wurzeln war, die sich hinreichend spezialisierte Kenntnisse anzueignen wußten. Mediziner traten auf diesem Gebiet hingegen kaum in Erscheinung, noch weniger freilich Allgemeinhistoriker oder selbst dokumentarische Papyrologen. Schon aufgrund ihrer besonderen fachlichen Anforderungen bilden die medizinischen Papyri jedenfalls meist eine Welt für sich.

So spielt auch ihre zeitliche und gesellschaftliche Verortung oft nur eine untergeordnete Rolle. Von Interesse war selbstredend die Frage der Überlieferung, insbesondere bei den Schriften der nachmals klassischen Autoren. ${ }^{1}$ Außerhalb dessen gerieten chronologische Fragen dagegen eher zur Nebensache, dies si-

\footnotetext{
* Nicola Reggiani und seinem Team habe ich sehr herzlich für die Einladung zu der Abschlußtagung des DIGMEDTEXT-Projekts zu danken und besonders dafür, daß sie mir dadurch den Anlaß dazu gaben, mich ein weiteres Mal und vor allem mit neuer Fragestellung auf dieses mir eher fernstehende Gebiet zu begeben. Der hier verfolgte Ansatz wurde im Rahmen des Heidelberger Sonderforschungsbereiches 933 Materiale Textkulturen. Materialität und Präsenz des Geschriebenen in non-typographischen Gesellschaften entwickelt und sollte sich, wie die folgenden Ausführungen zeigen, erneut als außerordentlich fruchtbar erweisen.
}

${ }^{1}$ Vgl. nur Marganne, Livre médical. 
cherlich auch deswegen, weil die Papyri allzu selten präzise Zeitangaben enthalten. Was sich an Datierungen in den Editionen findet, orientiert sich vorzugsweise an dem Erscheinungsbild der Schrift und ist also vor allem der Paläographie verpflichtet. Entsprechend sind eher Jahrhunderte als Jahrzehnte angegeben, wenn nicht manche Stücke sogar - etwa als 'byzantinisch' - einer ganzen Epoche zugewiesen werden. Entwicklungen zeichnen sich allenfalls insoweit ab, als man auf den ersten Blick bemerkenswerte Parallelen zu den eingangs beschriebenen 'Rangfragen' festzustellen meint. So scheinen in den Anfangszeiten noch medizinische Traktate zu überwiegen, doch steigt die Anzahl einzelner Rezepte im Verlauf der Kaiserzeit kontinuierlich an; zudem beginnen sich - so zumal bei den verschiedenen Arten von Fiebern - allmählich die Grenzen zwischen Magie und Medizin zu verwischen, bis in der Spätantike die magischen Aspekte womöglich sogar die Oberhand gewinnen.

Freilich bleibt noch zu fragen, wie weit dieser erste Eindruck auch die Wirklichkeit spiegelt und sich bei sorgfältiger Überprüfung der Evidenz am Ende tatsächlich zu erhärten vermag. Substantiellere Aussagen hierüber sind indessen nicht ohne weiteres zu treffen. Denn anders als die Texte selbst war ihre Gestalt oder besser ihre äußere Erscheinungsform weitaus seltener im Blick, wie offenbar auch nie der Frage nachgegangen wurde, ob es auch für diese Textsorte eine Art 'epigraphic habit', d.h. bestimmte bevorzugte Beschreibstoffe und Formate gab. Zwar lassen sich zu den Überlieferungsträgern viele wichtige Einzelbeobachtungen finden. Gleichwohl wirkt die Forschung zu den medizinischen Texten mehr noch als sonst vorzüglich an den Inhalten und allfälligen Erkenntnisfortschritten auf diesem Gebiet interessiert. Eine systematische Untersuchung der Formate scheint hingegen bislang unterblieben, und zwar erst recht, was die spätzeitliche Evidenz als ganze, also auch unabhängig von der jeweiligen Sprache, betrifft. Welch großes Versäumnis dies ist, hofft der vorliegende Beitrag deutlich zu machen. Dabei versteht sich von selbst, daß aus den genannten Gründen kaum mehr als ein erster, mit Hilfe von Stichproben gewonnener und daher noch sehr vorläufiger Überblick über das Material gewagt werden kann. Dennoch mag dies als Anstoß zu weiteren, zweifellos lohnenden Studien in dieser Richtung dienen.

Genauer wird es dabei um die Entwicklungen im medizinischen Schrifttum des kaiserzeitlichen und spätantiken Ägypten bis in das frühe Mittelalter hinein gehen, ${ }^{2}$ in dem neben dem Papyrus zunehmend andere Beschreibstoffe Bedeutung gewannen und auch Formate und Sprachen manche Wandlung erfuhren. $\mathrm{Da}$ es bei den Schriftträgern schon immer eine gewisse Bandbreite gab, sei dabei unbestritten, wie auch unter medizinischen Texten keineswegs nur die Schriften der großen Ärzte zu fassen sind, sondern neben der einschlägigen Fachliteratur stets auch Zeugnisse der Alltagspraxis standen. Damit sind wohl-

\footnotetext{
${ }^{2}$ Entsprechend handelt es sich bei den im folgenden genannten Daten, soweit nicht ausdrücklich anders angegeben, stets um solche der nachchristlichen Zeit.
} 
gemerkt nicht allfällige Nachrichten über akute Erkrankungen oder auch Behandlungsmethoden gemeint, wie sie sich in Privatbriefen, Votivinschriften, Orakelanfragen, ärztlichen Gutachten und dergleichen mehr finden, zumal dies stets - erst recht, wenn nur beiläufig eingestreut - den jeweils dort gebräuchlichen Formaten folgte. ${ }^{3}$ Vielmehr ist hier an nicht im strengen Sinne wissenschaftliche Texte zu denken wie die gerne im Wechselspiel von Frage und Antwort aufgebauten Lehrbücher oder sonstige Nachschlagewerke aller Art, wozu etwa auch die teilweise umfangreichen Rezeptsammlungen zählen. Für dieses gesamte Schrifttum war bis weit in die Kaiserzeit hinein bekanntlich die Papyrusrolle das typische Buchformat.

Anders sah dies freilich im Falle einzelner Rezepte aus, wofür erste Belege auf Ostraka bereits aus dem Neuen Reich und damit aus pharaonischer Zeit datieren. ${ }^{4}$ In ihrem großen Überblicksartikel über Papiri e scienza medica antica hatte denn auch schon Isabella Andorlini Marcone auf die zahllosen Belege von Rezepten, Arzneimittellisten und Etiketten auf Tonscherben sowie einzelnen Papyrus- oder Pergamentblättern hingewiesen, um eine heute in der Bodleiana aufbewahrte Gruppe von acht thebanischen Ostraka nochmals besonders hervorzuheben. ${ }^{5}$ Nur eine einzige dieser Scherbenaufschriften scheint bereits unter den Ptolemäern entstanden, wobei die Kombination mit einer geschäftlichen Notiz auf der anderen Seite gegen einen allzu professionellen Charakter der Aufzeichnung spricht. ${ }^{6}$ Ähnlich befremdlich erscheint das magische ablanathanalba auf der konkaven Seite eines dem I./II. Jhdt. zugeordneten Ostrakons aus Narmuthis. ${ }^{7}$ Dort wurde auch ein zwischen 150 und 225 entstandenes bilingues Inedi-

\footnotetext{
${ }^{3}$ Vgl. die von Jördens, Texte in Übersetzung zusammengestellten Beispiele, von denen hier lediglich die unter Nr. 9 angeführten Rezepte berücksichtigt werden, sowie die Tagungsbeiträge von A. Ricciar-

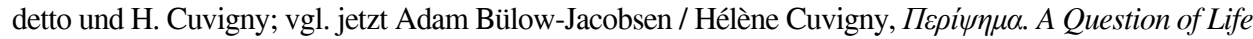
and Death in a Private Letter from the Eastern Desert of Egypt, unten S. 163-74.

${ }^{4}$ Vgl. Jonckheere, Prescriptions, und Westendorf, Handbuch, I, 59-65; zu ergänzen ist jetzt das von Hagen, Ostraca, 40-1 publizierte Kalksteinostrakon E.GA.6141.1943 (freundlicher Hinweis von J. F. Quack). Vgl. mit Abzeichnungen und Bildern auch M. Stuhr, Medizinische Schriften der Alten Ägypter, <http://www.medizinische-papyri.de/Start/index.html>.

${ }^{5}$ Andorlini Marcone, Apporto, das Zitat nach dem verkürzten Titel in der Kopfzeile; vgl. bes. S. 472 „Numerosissimi i frammenti di prescrizioni, ricette singole, elenchi di prodotti medicamentosi ed etichette di farmaci che ci provengono da papiri, ostraca e foglietti di pergamena ... Un

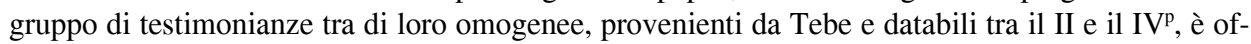
ferto dagli 8 ostraca della collezione della Bodleian Library continenti ricette per colliri, cicatrizzanti e pozioni collegabili con la migliore tradizione medica greca“; zu den letzteren bereits Préaux, Préscriptions. Berücksichtigt sind dabei allerdings lediglich O.Bodl. II 2181 bis 2188 (2181: II./III. Jhdt.; = TM\#63911. - 2182 = SB XIV 11708: II./III. Jhdt.; = TM\#63912. - 2183: IV. Jhdt.; = TM\#64502. - 2184: IV. Jhdt.; = TM\#64507. -2185 = SB XIV 117089: IV. Jhdt.; = TM\#64503. - 2186: IV. Jhdt.; = TM\#64504. - 2187: III. Jhdt.; = TM\#64214. - 2188: IV. Jhdt.; = TM\#64505), nicht jedoch der zweifelhafte Beleg in O.Bodl. II 2189 (IV. Jhdt.; = TM\#64506).

${ }^{6}$ O.Leid. 1 (II. Jhdt. v. Chr., Eleph.; = TM\#65613); vgl. auch Jördens, Texte, 340-1 Nr. 9.2.

${ }^{7}$ Suppl.Mag. II 67 B (I./II. Jhdt., Narm.; = TM\#92330).
} 
tum ergraben, vier weitere Ostraka - aus Elephantine und Theben bzw. unbekannter Herkunft - sind in das II. Jhdt. oder nur allgemein in die römische Zeit gesetzt. ${ }^{8}$ Die Mehrzahl der Zeugnisse datiert jedoch deutlich später - so namentlich die koptischen Texte - und stammt zudem durchweg aus Oberägypten, genauer noch, sieht man von einem weiteren Ostrakon aus Elephantine und einem Einzelstück aus dem Wadi Sarga ab, aus der thebanischen Region. ${ }^{9}$

Darunter fallen auch einige Kalksteinostraka, ${ }^{10}$ worin zweifellos eine Art ,epigraphic habit' zu erkennen ist, da das in dieser Weise genutzte Gestein für das Westufer typisch ist. Zwar könnte die Verwendung von Tonscherben überhaupt auf oberägyptische Vorlieben verweisen, doch sind reiche Ostrakafunde auch aus dem Faijum, etwa aus Philadelpheia, Karanis oder Soknopaiu Nesos bekannt. Wenn im gesamten Unter- und Mittelägypten bisher nur in Narmuthis zwei medizinische Ostraka zutage traten, wird man die Erklärung demnach wohl eher in der frühen Zeitstellung unserer dortigen Evidenz zu suchen haben.

Chronologische Gründe sind in jedem Fall bei dem späten und zudem relativ seltenen Aufkommen medizinischer Texte auf Pergament zu vermuten. ${ }^{11}$ Das früheste Zeugnis scheint bislang ein kleines Fragment mit wenigstens drei Rezepten aus dem mittleren III. Jhdt. zu sein, das nur auf der Haarseite Schrift trägt

${ }^{8}$ OMM inv. 155 (150-225, Narm.; = TM\#63578); O.Stras. I 619 (II. Jhdt., Theben; = TM\#63705); O.Leid. 2 (röm. Zeit, Eleph.; = TM\#63311); kaiserzeitlich sind zudem das demotische O.Leid.Dem. 334 (= TM\#49360, Theben; die Einordnung als medizinisch gegen Ritner, $M e$ chanics, bes. S. 69 Anm. 311 nochmals bestätigt von Quack, Kontinuität, 77 Anm. 2) sowie das hieratische Ostrakon O.Berol. 5570 (Herk. unbek.; = TM\#89695, mit Jonckheere, Prescriptions, 56 ff.). Noch spärlicher ist die pharaonenzeitliche Evidenz, da es außer den in Anm. 10 genannten thebanischen Kalksteinostraka offenbar höchstens zwei weitere Tonscherben medizinischen Inhalts gibt, vgl. zuletzt Westendorf, Handbuch, I, 60-1 mit O. London Amarna 297 (Nr. 1, unsicher) sowie O.Louvre E 3255 (Nr. 2).

${ }^{9}$ So außer einigen der bereits in Anm. 5 genannten Ostraka der Bodleiana auch O.Bodl. II 2564 descr. (IV.-VI. Jhdt.; = TM\#64660) und O.Mon.Epiph. II 622 (VI./VII. Jhdt.; = TM\#65226); zu den koptischen Texten jetzt Richter, Texte, 189 mit insgesamt sieben Ostraka sowie dem irrigerweise als Nr. 16 unter den Papyri eingeordneten O.WadiSarga 20 (V.-VII. Jhdt.; = TM\#108461). Zu ergänzen sind O.Brit.Mus.Copt. I, pl. 83.3 (VI.-VIII. Jhdt.; = TM\#111821), vielleicht auch pl. 83.4 (VII./VIII. Jhdt.; = TM\#82819, Elephantine), O.Frangé 101 (VIII. Jhdt.; = TM\#141026) sowie die offenbar weiterhin unedierten, größtenteils im thebanischen Antikenhandel erworbenen Tonscherben O.Berol. 4991, 5151, 5163, 17973 sowie 19902, vgl. Beltz, Katalog, II, 170 Nr. IV 1021-1026.

${ }^{10}$ Nach Richter, Texte, 189 die sämtlich dem VII./VIII. Jhdt. zuzuordnenden BKU I 27 (= TM\#704843, vgl. auch Beltz, Katalog, II, 170 Nr. IV 1020) und 28 = SB Kopt. II 1029 (= TM\#85160, vgl. auch Beltz, Katalog, II, 170 Nr. IV 1019), O.Brit.Mus.Copt. I, pl. 49.1 (= TM\#111820), II 37 (= TM\#82903) sowie O.Crum 487 (= TM\#83376); vgl. auch die jetzt insgesamt sechs bereits pharaonenzeitlichen Ostraka aus Deir el-Medineh mit Westendorf, Handbuch, bes. I, $61 \mathrm{ff}$. Nr. 3.a-e sowie den bereits in Anm. 4 erwähnten neuen Beleg aus dem Fitzwilliam Museum. Erwähnung verdient auch der vermutlich als Votivgabe im damaligen Amenotes-Heiligtum niedergelegte, vielfach behandelte Bericht des Polyaratos über seine wunderbare Heilung I.Deir el-Bahari A 1 (1./2. 260 v. Chr.; = TM\#6312), der ebenfalls auf einem Kalksteinostrakon festgehalten wurde.

${ }^{11}$ Hierzu zuletzt Marganne, Hippocrate, bes. S. 292. 
und wohl ein Einzelblatt sein dürfte $;^{12}$ sicher ist dies bei einem hermopolitischen Stück aus dem frühen IV. Jhdt., wie die an drei Seiten erhaltenen originalen Ränder und vor allem die Faltungen anzeigen..$^{13}$ Rezepte liegen auch auf drei Codexfragmenten des V. bzw. V./VI. Jhdts. vor. ${ }^{14}$ Noch unediert scheint ein koptischer medizinischer Text aus einem Codex wohl des VI. Jhdts. zu sein, der sich auf einem Doppelblatt der Berliner Papyrussammlung findet. ${ }^{15}$ Erwähnt sei auch ein aus dem V./VI. Jhdt. stammendes Etikett, das den Editoren zufolge ,,als Beschriftung für einen Behälter (diente) ${ }^{\prime} .{ }^{16}$ Freilich ist aus der ein- oder zweiseitigen Beschriftung allein noch keine Sicherheit über die Zugehörigkeit zu einem Codex zu erlangen. So blieb bei dem neuen oxyrhynchitischen Fragment die Fleischseite zwar unbeschrieben, doch lassen die rote Rahmung des Schriftspiegels und die gleichmäßige Breite der Freiränder eher an einen Codex denken, während ein Bruchstück unbekannter Herkunft mit vier Rezepten auf Vorderund Rückseite nur ein Notizzettel gewesen sein mag. ${ }^{17}$

Was die sonstigen Schriftträger betrifft, stammen sämtliche erhaltenen Belege aus dem koptischen Bereich, nämlich außer fünf medizinischen Texten auf Papier auch die beiden Dipinti, die am Wadi Sarga und dem Jeremiaskloster in Saqqara auf Wandverputz aufgetragen wurden. ${ }^{18}$ Dabei ist ersteres sicher rein zeitlich zu deuten, während letzteres dem Zufall der Überlieferung geschuldet sein mag. Weitaus mehr muß hingegen erstaunen, daß medizinische Texte offenbar niemals auf Holz niedergelegt wurden, jedenfalls nicht in Ägypten. Denn die einzige Liste mit Substanzen, die zu einem Rezept gehören könnten, wurde in Vindolanda gefunden. ${ }^{19}$ Daran bestätigt sich letztlich nur, daß man zu dem nächstgelegenen Schreibuntergrund griff, um solche Zusammenstellungen festzuhalten, wie wir sie in Ägypten eben auf Einzelblättern oder Ostraka finden. Offenbar wollte man sich auf diese Weise aus akutem Anlaß ein geeignetes Mittel notieren, gegebenenfalls auch je nach Verfügbarkeit der Substanzen mehrere Varianten zur Auswahl mit

${ }^{12}$ P.Ryl. I 29 (III. Jhdt.; = TM\#64106).

${ }^{13}$ SB XXVI 16458 = PSI VI 718 (frühes IV. Jhdt.; = TM\#64564).

${ }^{14}$ P.Horak 14 (V. Jhdt.; = TM\#168982), MPER N.S. XIII 8 (V. Jhdt.; = TM\#64793) und wohl auch BKT III 32 f. (V./VI. Jhdt.; = TM\#64855), bei dem es sich grundsätzlich auch um ein beidseitig beschriebenes Einzelblatt handeln könnte.

${ }^{15}$ P.Berol. 5530, vgl. Beltz, Katalog, I, 108 Nr. III 11.

${ }^{16}$ MPER N.S. XIII 11 (V./VI. Jhdt.; = TM\#107409).

${ }^{17}$ P.Oxy. LXXX 5251 (III./IV. Jhdt.; = TM\#388557) bzw. MPER N.S. XIII 12 (VI./VII. Jhdt.; = TM\#65276; unter M-P3 02422.500 in der online Database als ,codex parchemin“ klassifiziert, vorsichtiger dagegen Andorlini Marcone, Apporto, 544 Nr. 202 „Fr. di pergamena“).

${ }^{18}$ Nach Richter, Texte, bes. S. 191 auf Papier: SB Kopt. I 2 und 5 (beide X./XI. Jhdt; = TM\#101349 bzw. 101352), P.Ryl.Copt. 104 und 106 (beide XI. Jhdt; = TM\# 98059 bzw. 111284) sowie BKU I 26 (XI. Jhdt; = TM\#704842; vgl. auch Beltz, Katalog, I, 105 Nr. II 40); auf der Wand: O.WadiSarga 21 (VII./VIII. Jhdt; = TM\#371976) sowie der von H. Thompson bei Quibell, Excavations, 57 Nr. 103 (o.D.) publizierte ,extract from a collection of medical prescriptions“ in Raum 700 D des Jeremiasklosters.

${ }^{19}$ T.Vindol. III 591 (etwa 92-115; = TM\#130224). 
sich führen. Eine längerfristige Aufbewahrung, wie sie dem bevorzugten Einsatz von Holztafeln im Schulwesen, aber auch bei Vertragsformularen zugrundelag, scheint dagegen nicht beabsichtigt gewesen zu sein.

Kehren wir damit von der Aufzeichnung einzelner Rezepte zu größeren Sammlungen derartiger Texte, zu Nachschlagewerken und wissenschaftlichen Traktaten zurück und der Frage, ob es bei medizinischen Schriften bestimmte bevorzugte Buchtypen gab. Da es sich um eine erste Sondierung handelt, die auch methodisch Neuland betritt, sei zunächst von der üblichen Binnendifferenzierung nach Genera abgesehen und stattdessen mit einem gemischten, aber überschaubaren Textcorpus begonnen, das als eine Art Stichprobe willkürlich herausgegriffen sei. Als geeignetes Untersuchungsobjekt bieten sich die kürzlich in P.Oxy. LXXX publizierten Zeugnisse aus Oxyrhynchos an. Obwohl sämtlich erst in nachchristlicher Zeit entstanden, stellen die insgesamt 35 medizinischen Texte - ohne die ärztlichen Gutachten wohlgemerkt - eine beträchtliche Erweiterung unserer Materialbasis dar, bedeutet doch allein dies einen Zuwachs von rund 15\% gegenüber den 224 Zeugnissen, die Isabella Andorlini Marcone im Jahr 1993 aufzulisten vermochte, selbst wenn in zwei Fällen nur Ergänzungen zu bereits edierten Texten geboten werden. ${ }^{20}$ Dabei treffen wir auf eine relativ traditionelle Buchkultur, wie die nahezu ausschließliche Verwendung von Papyrus als Schreibmaterial anzeigt; von dem einzigen Pergamentfragment darunter war bereits oben die Rede.

Bis in das III. Jhdt. hinein haben wir danach - fast möchte man sagen: erwartungsgemäß - mit Rollen zu tun; ebenso unmittelbar findet mit dem ausgehenden III. und beginnenden IV. Jhdt. der Übergang zum neuen Format des Codex statt. ${ }^{21}$ Im Fall von zwei Rezepten, die von unterschiedlicher Hand auf Vorderund Rückseite desselben Papyrus festgehalten wurden, ist unklar, wie das Original einst aussah. ${ }^{22}$ Nur zwei Mal handelt es sich sicher um Einzelblätter, beides Listen von Ingredienzien im Genetiv mit Gewichtsangabe, die dem V. bzw. VI. Jhdt. zugeordnet werden. Während das spätere Fragment unter optimaler Ausnutzung der Schreibfläche fast von Rand zu Rand beschrieben ist, jedoch eine freie Rückseite besitzt, befindet sich die frühere Liste auf dem Verso einer umfangreicheren Aufstellung von Weinlieferungen, die zu diesem Zwecke eigens zurechtgeschnitten wurde. ${ }^{23}$ Beide wird man insofern den oben erörterten Ostraka an die Seite stellen dürfen.

Um so überraschender ist indes, daß wir ganz ähnliche Formen der Zweitverwendung auch bei zahlreichen anderen Texten des Bandes antreffen, und zwar

\footnotetext{
${ }^{20}$ Nämlich bei P.Oxy. LXXX 5221 + P.Köln VII 311 (III. Jhdt., bislang unbekannter Herkunft; = TM\#60182) sowie P.Oxy. LXXX 5235 + P.Mil.Vogl. I 15 (IV. Jhdt.; = TM\#64473), bei Andorlini Marcone, Apporto, 548 Nr. 22.1 bzw. 516 Nr. 103.

${ }^{21} \mathrm{Vgl}$. auch bereits Marganne, Livre médical, 24-5.

${ }^{22}$ P.Oxy. LXXX 5249 und 5250 (III. Jhdt.; = TM\#388555 bzw. TM\#388556).

${ }^{23}$ P.Oxy. LXXX 5253 (VI. Jhdt.; = TM\#388559) sowie 5252 (V. Jhdt.; = TM\#388558).
} 
selbst bei den bekannten Medizinschriftstellern. So blieb lediglich bei acht der insgesamt 26 Papyri im Rollenformat die Rückseite gänzlich unbeschrieben; in drei weiteren Fällen finden sich jeweils nur wenige Zeilen, deren Zusammenhang mit dem Text auf der Vorderseite unklar ist. ${ }^{24}$ Zwölf Texte und damit nahezu die Hälfte der Schriften wurde hingegen auf der Rückseite von Verwaltungsakten niedergelegt, die zumeist nur allgemein als ,account“, manchmal aber auch als „two columns of cursive“, „documentary text", „tax roll“" oder „register" gekennzeichnet sind; das Rezept eines Iulianus aus Caesarea gegen Zahnprobleme fand sogar auf dem Verso eines unter den Philippi gefertigten Dokuments der lateinischsprachigen Militärverwaltung Platz. ${ }^{25}$ Einen Sonderfall stellt eine auf beiden Seiten von derselben Hand beschriebene Rolle aus dem I./II. Jhdt. dar, auf deren Rekto sich ein griechisch-lateinisches Glossar in griechischen Buchstaben befindet; ebenso ungewöhnlich ist die vom Schreiber vorgenommene Drehung um $180^{\circ}$, um den Kommentar zum ersten Buch von Hippokrates' Epidemiae auf dem Verso niederzulegen. ${ }^{26}$

Dies ist ein durchaus bemerkenswerter Befund, der sich jedoch an den insgesamt elf Papyri aus der frühen und vor allem hohen Kaiserzeit bestätigt, die wenige Jahre zuvor in P.Oxy. LXXIV vorgelegt worden waren. Obwohl nicht einmal ein Drittel des jetzt in P.Oxy. LXXX gesammelten Materials, hatte dies seinerzeit die bis dahin in dieser Reihe publizierte Menge medizinischer Texte auf einen Schlag verdoppelt und kann insofern in jedem Fall als willkommene Gegenprobe dienen. Bei vier dieser elf Papyri haben wir es mit Rezepten auf Einzelblättern zu tun, wobei selbst hier gleich zwei davon eine Beschriftung von zweiter, kursiverer Hand auf der anderen Seite tragen. ${ }^{27}$ Bei den sonstigen Texten stellt dies sogar die Regel dar. Denn nur bei dem frühesten, vielleicht noch

${ }^{24}$ So bei P.Oxy. LXXX 5220 (II./III. Jhdt.; = TM\#388528), 5222 (II./III. Jhdt.; = TM\#388529), 5236 (II./III. Jhdt.; = TM\#388542), 5237 (II. Jhdt.; = TM\#388543), 5238 (II./III. Jhdt.; = TM\#388544), 5239 (II./III. Jhdt.; = TM\#388545), 5240 (I. Jhdt.; = TM\#388546) und 5245 (II. Jhdt.; = TM\#388551); bei 5223 (I. Jhdt; = TM\#388530) und 5225 (II. Jhdt.; = TM\#388532) sind noch Reste zweier Zeilen auf dem Verso zu erkennen, bei 5230 (III. Jhdt.; = TM\#388555) weist lediglich das Verso von fr. 3 Schrift auf.

${ }^{25}$ So jeweils in der Einl., vgl. ,account“: P.Oxy. LXXX 5221 (+ P.Köln. VII 311; III. Jhdt.; = TM\#60182), 5241 (II./III. Jhdt.; = TM\#388547), 5242 (II. Jhdt.; = TM\#388548), 5243 (II./III. Jhdt.; = TM\#388549), 5247 (II./III. Jhdt.; = TM\#388553), 5248 (II./III. Jhdt.; = TM\#388554, hier kopfstehend); „two columns of cursive“: 5226 (III. Jhdt.; = TM\#388533); „,documentary text“: 5232 (II./III. Jhdt.; = TM\#388539), 5234 (II. Jhdt.; = TM\#388541); ,tax roll“: 5233 (II./III. Jhdt.; = TM\#388540); ,register“: 5246 (II./III. Jhdt.; = TM\#388552); ,Latin military document“: 5244 (III. Jhdt.; = TM\#388550).

${ }^{26}$ P.Oxy. LXXVIII 5162 bzw. LXXX 5231 (beide I./II. Jhdt.; = TM\#171907 bzw. TM\#388538).

${ }^{27}$ Nur einseitig beschriftet sind von den durchweg auf das II./III. Jhdt. datierten Blättern P.Oxy. LXXIV 4978 (= TM\#119323) und 4979 (= TM\#119324); von einer größeren Rolle stammt dagegen der Einl. zufolge 4976 (= TM\#119321; ,,The front contains scant remains of two columns of a document. The papyrus was pre-cut to accommodate the text on the back"), während sich der Sachverhalt bei 4977 (= TM\#119322) schon wegen der ungewöhnlichen Abschrift eines weiteren Rezeptes auf dem linken Rand komplexer darstellt. 
im I. Jhdt. entstandenen Traktat griff man zu einer frischen Rolle; der schon hieraus ersichtliche Anspruch wird durch die saubere Schrift und den großzügigen oberen Rand weiter gestützt. ${ }^{28}$ Dagegen kam in allen anderen Fällen, auch wenn die Beschriftung teilweise kaum weniger sorgfältig ausfiel, erneut bereits anderweitig verwendetes Schreibmaterial zum Einsatz, vorzugsweise auch hier wieder offenkundig ausgesonderte Verwaltungsakten. ${ }^{29}$

Der einzige Text eines bekannten Autors unter diesen elf Papyri kann insofern um so mehr Aufmerksamkeit verlangen, da der aus zwei verschiedenen Grabungskampagnen hervorgegangene P.Oxy. LXXIV 4969 nicht nur den ersten antiken Zeugen von Hippokrates' Schrift De articulis enthält, sondern sich schon um die Wende des II. zum III. Jhdt. in Codexform präsentiert. ${ }^{30}$ Damit zählt er zu unseren frühesten Codices überhaupt, womit wir wieder zu unserem eigentlichen Untersuchungsgegenstand P.Oxy. LXXX zurückkehren. Auch bei den restlichen, zunächst zurückgestellten sechs Papyri handelt es sich um Codices, worunter sich ebenfalls recht frühe Vertreter finden. Bereits in das III./IV. Jhdt. wird etwa außer dem schon genannten Pergament auch das kleine Fragment mit Hippokrates' Aphorismen V und VI gesetzt. ${ }^{31}$

Trotz seiner geringen Größe von gerade einmal 3,5 x 2,7 cm lassen sich in diesem Fall sogar genauere Aussagen über das Codexformat treffen. Denn bei einer Zeilenbreite von \pm 29 Buchstaben sollte der überlieferte Text rund 150 Zeilen eingenommen haben, so daß mit jeweils zwei Spalten auf der Seite zu rechnen ist. Parallelen hierfür gibt es durchaus. ${ }^{32}$ Mehr Beachtung ist insofern dem Umstand zuzumessen, daß gleich die Hälfte der in P.Oxy. LXXX vorgelegten Papyruscodices dieses Layout besitzt, zumal ihre zeitliche Streuung vom III./IV. bis zum V./VI. Jhdt. gegen eine reine Modeerscheinung spricht. In allen drei Fällen sind überdies Schriften der großen Ärzte betroffen, nämlich außer den bereits genannten Aphorismen des Hippokrates auch die ersten Bücher von Dioskurides' De materia medica und Galens De locis affectis. ${ }^{33}$ Keine Besonderheiten scheinen hingegen bei den verbleibenden drei Papyruscodices zu vermerken, einem „Repetitorium für Studierende der Medizin“ aus dem IV. Jhdt. ${ }^{34}$ so-

\footnotetext{
${ }^{28}$ P.Oxy. LXXIV 4971 (I./II. Jhdt.; = TM\#119316).

${ }^{29}$ P.Oxy. LXXIV 4970 (II. Jhdt.; = TM\#119317): „on the back of a land register“; 4973 (II. Jhdt.; = TM\#119318): „on the back of a register of landholdings and taxes“; 4975 (II. Jhdt.; = TM\#119320): „on the back of a document“ sowie 4972 (II./III. Jhdt.; = TM\#119315): „on the back of a letter“. Bei dem ebenfalls gegen die Fasern beschriebenen P.Oxy. LXXIV 4974 (II./III. Jhdt.; = TM\#119319) bleibt letztlich unklar, ob der partiell abgewaschene Tintenfleck auf der anderen Seite mit einer Vorbeschriftung zu verbinden ist.

${ }^{30}$ P.Oxy. LXXIV 4969 (II./III. Jhdt.; = TM\#119314).

${ }^{31}$ P.Oxy. LXXX 5251 (III./IV. Jhdt.; = TM\#388557; vgl. bereits oben Anm. 17) bzw. 5219 (III./IV. Jhdt.; = TM\#388527).

${ }^{32} \mathrm{Vgl}$. nur Turner, Typology, $35 \mathrm{ff}$.

${ }^{33}$ P.Oxy. LXXX 5224 (IV. Jhdt.; = TM\#388531) bzw. 5227 (V./VI. Jhdt.; = TM\#388534).

${ }^{34}$ So K. Deichgräber in der Ed. pr. des bereits 1937 publizierten P.Mil.Vogl. I 15, der jetzt
} 
wie zwei weiteren Werken Galens aus dem VI. Jhdt. ${ }^{35}$

Faßt man die aus unseren beiden Stichproben P.Oxy. LXXIV und LXXX gewonnenen Ergebnisse zusammen, weist das medizinische Schrifttum in Oxyrhynchos abgesehen von den wenigen Einzelblättern doch einige unerwartete Züge auf. Dabei überrascht weniger, daß der Codex gegen Ende des III. Jhdts. an die Stelle des bis dahin üblichen Rollenformats tritt, wiewohl Papyrus weiterhin der gängige Beschreibstoff bleibt. Erstaunlicher ist vielmehr der hohe Prozentsatz an wiederverwendeten Rollen, die zumindest teilweise aus öffentlichen Beständen stammen. Zwar stellt der großzügige Umgang der Verwaltung mit den Ressourcen eine wohlfeile Erklärung für den Einsatz jungfräulicher Rückseiten zu derartigen Zwecken dar, doch ist eine gewisse Systematik kaum zu bestreiten. Beachtung verdient überdies die relativ große Anzahl zweispaltiger Codices.

Freilich ist keineswegs klar, wie repräsentativ diese Befunde für das medizinische Schrifttum in Ägypten als ganzes sind. Insofern bietet sich eine Gegenüberstellung mit der Evidenz aus Antinoupolis an, das nicht zuletzt wegen seines Lokalheiligen, des Arztes Kolluthos, geradezu als Hochburg der ägyptischen Medizin galt, ${ }^{36}$ wenngleich die 28 medizinischen Texte nicht im Umfeld seiner Orakelstätte zutage traten. ${ }^{37}$ Die ersten Zeugnisse setzen schon bald nach der Gründung der Stadt im Jahr 130 ein, um sich dann ohne größere Unterbrechung bis zum VII. Jhdt. zu erstrecken. ${ }^{38}$ Die hierdurch bewirkte zeitliche Verschiebung bietet zweifellos die Erklärung, warum wir anders als in Oxyrhynchos fast ausschließlich mit Codices zu tun haben; nur ein noch dem II./III. Jhdt. zugewiesener Text wurde noch auf eine Rolle geschrieben, während die ebenfalls unbeschriftete Rückseite bei einem Fragment des V./VI. Jhdts. eher an ein Einzelblatt denken ließ. ${ }^{39}$ Bemerkenswerter erscheint auf den ersten Blick der relativ hohe Anteil von immerhin drei Pergamentcodices; ${ }^{40}$ da in Oxyrhynchos ein Codex aus Pergament auf sechs aus Papyrus kam und es hier vier von insgesamt 26 sind, erweist sich das Verhältnis bei näherer Betrachtung aber gar nicht als so unterschiedlich.

Wichtiger erscheint demgegenüber, daß sämtliche Pergamentcodices zu den frühesten Zeugnissen für dieses Genos zählen, wobei die Überlieferung über die Jahrhunderte hinweg keineswegs gleichmäßig fließt. Denn trotz aller Kontinuität blieben selten mehr als ein oder zwei medizinische Texte erhalten, bis das Mate-

durch P.Oxy. LXXX 5235 ergänzt werden konnte (IV. Jhdt.; = TM\#64473).

${ }^{35}$ P.Oxy. LXXX 5228 und 5229 (VI. Jhdt.; = TM\#38853 bzw. TM\#388536).

${ }^{36}$ Vgl. nur Marganne, Collection; zu den Fundorten auch Menci, Antinoe, 51-2; Fournet, Antinoupolis, 129 ff. sowie zuletzt Del Corso/Pintaudi, Papiri, 7-8.

${ }^{37}$ So jetzt bes. Fournet, Antinoupolis, bes. S. 128-9.

${ }^{38} \mathrm{Vgl}$. nur Menci, Antinoe, bes. S. 51.

${ }^{39}$ P.Ant. III 138 (II./III. Jhdt.; = TM\#63874) bzw. 137 (V./VI. Jhdt.; = TM\#64900) mit der offenbar hierauf zu beziehenden Einordnung „1 $f$ (oglio)? $p$ (apiraceo)“ unter den Rubriken $V$-VI und Medicina in der Tabelle von Menci, Antinoe, 51.

${ }^{40}$ P.Ant. I 28 (III./IV. Jhdt.; = TM\#60189); PSI Ant. inv. 320 A (III. Jhdt., vgl. Del Corso/Pintaudi, Papiri, 10-17 Nr. 3; = TM\#642455); P.Ant. III 133 (III./IV. Jhdt.; = TM\#64330). 
rial im VI. Jhdt. plötzlich sprunghaft ansteigt und sogar zweistelligen Umfang erreicht. Aus dieser Zeit stammen auch die fünf antinoitischen Belege für Schriften des Galen und besonders Hippokrates, der sonst nur noch in einem Pergamentcodex aus dem III./IV. Jhdt. begegnet. ${ }^{41}$ Während die letzteren im Corpus dei papiri filosofici nochmals eine eingehende Behandlung erfuhren, wurde den anderen antinoitischen Stücken nur selten größere Aufmerksamkeit zuteil. So hatte sich schon der Herausgeber J. W. B. Barns zumal bei den unscheinbareren Fragmenten mit relativ spärlichen Hinweisen und eher allgemeinen Bemerkungen zur Qualität von Material und Schrift begnügt, zwar immer sorgfältig Maße und Faserverlauf bzw. die Zuordnung von Haar- und Fleischseite notiert, aber selbst für die Datierung in der Regel keinerlei Begründung gegeben. Die einzige Ausnahme bilden die ansprechenden Überlegungen von Federico Morelli zu dem kaum mehr als 50 Buchstaben umfassenden, heute allerdings verlorenen Frg. 1 von P.Ant. III 139, nur daß der von ihm vermutete direkte Anschluß an Frg. 10 des Galencodex nicht mehr zu verifizieren ist. ${ }^{42}$ So mag zwar anders als in Oxyrhynchos bisher noch kein zweispaltiger medizinischer Codex aus Antinoupolis nachweisbar sein, doch könnte eine nochmalige Überprüfung der oft schmalen Bruchstücke vielleicht Neues dazu erbringen.

Mit der antinoitischen Buchkultur als ganzer hatte Giovanna Menci sich anläßlich der Hundertjahrfeier der dortigen Grabungen befaßt und besonders die Unterschiede in der Wahl des Beschreibstoffes hervorgehoben: Während der Anteil der Pergamentcodices bei Adespota lediglich 10\% betrage, liege er bei bekannten Autoren drei Mal so hoch, zudem handele es sich stets um relativ wertvolle Exemplare. ${ }^{43}$ In dem hier in Rede stehenden Bereich scheint sich diese Beobachtung freilich nicht bestätigen zu lassen. Gewiß fällt ein Pergamentcodex bei insgesamt nur sechs Schriften der großen Ärzte schwerer ins Gewicht als drei unter 22 noch nicht identifizierten Werken, doch sind die Differenzen im Grunde gering und können sich mit jedem Neufund erheblich verschieben, was letztlich auf die viel zu schmale Materialbasis für derlei prozentuale Rechnungen verweist. Zudem handelt es sich keineswegs immer um Luxusausgaben, abge-

${ }^{41}$ Galen: P.Ant. III $139+186$ (hierzu auch die folgende Anm. mit Text; = TM\#59961); Hippokrates: P.Ant. III 183; 184; 185 (sämtlich VI. Jhdt.; = TM\#60191, 60192 und 60190); P.Ant. II 86 (VI./VII. Jhdt.; = TM\#60193) sowie P.Ant. I 28 (III./IV. Jhdt., mit Cavallo, Calamo, $18=$ TM\#60189); vgl. auch die Tabelle bei Menci, Antinoe, 50. Auf die teilweise abweichenden Datierungen in CPF I.2 14 (Galen) bzw. 18 (Hippokrates; so bes. S. 78 in der Einl. von D. Manetti zu P.Ant. I 28 = F 1 mit einer Datierung in das V. Jhdt.) sei lediglich hingewiesen.

${ }^{42} \mathrm{Vgl}$. nur Morelli, P. Ant. III 139.

${ }^{43}$ So Menci, Antinoe, 52. Bei der Tabelle der Testi adespoti (profani) auf S. 51 ist der Eintrag ,$c$ (odice) $p$ (apiraceo)“ unter den Rubriken III-IV und Medicina allerdings ohnehin in , $c$ (odice) $m$ (embranaceo)“ zu ändern, da es sich um P.Ant. III 133 (III./IV. Jhdt.; = TM\#64330) handeln dürfte; unter dem III. Jhdt. wären jetzt zudem zu ergänzen die beiden kürzlich publizierten PSI Ant. inv. NN 16-12-2013 (Anf. III. Jhdt., $c p$; vgl. Del Corso/Pintaudi, Papiri, 8-13 Nr. 2; = TM\#642454) sowie PSI Ant. inv. 320 A (2. Hälfte III. Jhdt, cm; ebda. S. 10-17 Nr. 3; = TM\#642455). 
sehen davon, daß mit besonderer Sorgfalt gefertigte Exemplare durchaus auch unter Papyruscodices begegnen; erinnert sei nur an das sog. Antinoopolis Illustrated Herbal mit seinem durchweg waagerechten Faserverlauf. ${ }^{44}$

In der Regel haben wir es vielmehr mit Gebrauchstexten zu tun, wie auch schon Giovanna Menci eine gewisse Vorliebe der Antinoiten für technische und wissenschaftliche Spezialliteratur festgestellt hatte. ${ }^{45}$ Doch gilt dies möglicherweise für das medizinische Schrifttum ganz allgemein. Denn hier wird man auch die rote Rahmung des oxyrhynchitischen Pergaments einordnen wollen, die bereits die Herausgeber mit der entsprechenden Praxis bei astronomischen Werken in Verbindung brachten. ${ }^{46}$ In eine ähnliche Richtung dürfte die so häufige und sicherlich kostengünstige Zweitverwendung bereits beschriebener Rollen weisen. Ein noch wichtigeres Indiz aber ist in dem oben beobachteten Einsatz zweispaltiger Codices zu sehen. Erst kürzlich hat Jean-Luc Fournet darauf aufmerksam gemacht, daß die Mehrzahl der einschlägigen Zeugnisse außerhalb der christlichen Literatur den großen Rednern galt, weswegen auch Thukydides vornehmlich wegen der in sein Werk eingebetteten Reden in diesem Format Platz gefunden haben dürfte. ${ }^{47}$ Die beiden einzigen Ausnahmen bildeten bisher zwei Papyruscodices des III. Jhdts. mit der astrologischen Dichtung des Anubion und erneut einem medizinischen Werk, nämlich einem dem Heliodor zugewiesenen Traktat über die Behandlung einer gebrochenen Schulter. ${ }^{48}$ Worin immer die Gründe für diese Gestaltung zu suchen sind - vielleicht in einer leichteren Memorierbarkeit $-{ }^{49}$ scheint dieses Format demnach vorzüglich den Bedürfnissen des gehobenen Unterrichts und der anspruchsvolleren (Aus-)bildung zu entsprechen.

Im übrigen sind Codices bekanntlich nicht gleich Codices. Während zumeist

${ }^{44}$ Leith, Herbal, bes. S. 142, demzufolge bei dem zwischen 350 und 450 entstandenen Papyruscodex (= TM\#64598) jeweils zwei Blätter mit ihren Außenseiten aufeinandergeklebt wären. Allerdings bleibt unklar, ob auch die Möglichkeit einer Spezialanfertigung in der Art der aus drei Lagen bestehenden Protokolla geprüft wurde.

${ }^{45} \mathrm{Vgl}$. nur Menci, Antinoe, bes. S. 49: „Non a caso vediamo in Antinoe [...] quanto fossero diffusi gli interessi per le attività tecnico-pratiche, le discipline tecnico-scientifiche, il genere letterario manualistico“, vgl. auch S. 51: „La presenza di numerosi testi di carattere scientifico e tecnicopratico, in forma prevalentemente manualistica, è tipica di Antinoe ed è certamente legata a scuole specialistiche, di cui, fra l'altro, abbiamo anche attestazioni archeologiche o letterarie“.

${ }^{46}$ Vgl. nur M. Hirt, Einl. zu P.Oxy. LXXX 5251: „,The use of a red border is unusual, but cf. the papyrus codex LXI 4163 (template for the sun and procedure text [III. Jhdt.; = TM\#65962]), and in general the use of red ruling in tables (e.g. A. Jones, Astronomical papyri from Oxyrhynchus (1999) passim).“

${ }^{47}$ Fournet, Lambeaux, 62-3, wozu auch der jetzt in P.Oxy. LXXX als Parallele benannte P.Oxy. LXXIII 4949 (VI. Jhdt.; = TM\#117828) mit Aelius Aristeides' Panathenaikos paßt. Anders dagegen noch Dorandi, Tradierung, demzufolge die Zweispaltigkeit „bei Büchern mit Dichtung“ (S. 9) zu beobachten sei.

${ }^{48}$ P.Oxy. LXVI 4503 (= TM\#59140) bzw. P.Ryl. III 529 (= TM\#59970).

${ }^{49}$ Vgl. auch schon Fournet, Lambeaux, bes. S. 63-4 mit Überlegungen in Richtung der kognitiven Psychologie. Schmale Kolumnen zeichneten Rednertexte bereits im Rollenformat aus, vgl. nur Dorandi, Tradierung, 6. 
nur die Anordnung der Lagen und des Faserverlaufs in den Blick genommen wird, wurde zumal bei medizinischen Texten gerne auch auf die nach Galen in

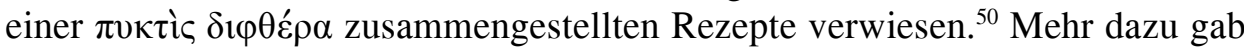
jetzt seine kürzlich wiederentdeckte Schrift über den rechten Umgang mit Verlusterfahrungen preis, in der er seine Reaktion auf die Vernichtung all seiner Medikamente, Geräte und Bücher im Feuer des Jahres 192 beschreibt, worunter der Verlust seiner Rezeptsammlungen oder kurz $\delta i \varphi \theta \varepsilon ́ \rho \alpha \iota$ das bitterste sei. ${ }^{51}$ Selbst bei den beiden wertvollen Exemplaren, derer Galen dort mit besonderer Wärme gedenkt, kann es sich freilich kaum um Bücher in einem festen Einband gehandelt haben. ${ }^{52}$ Denn zum einen waren sie offenbar über Jahrzehnte hinweg angelegt worden, zum anderen muß es bei derartigen Handbüchern nicht zuletzt darum gegangen sein, weitere Rezepte jederzeit am richtigen Ort hinzufügen zu können.

Folgerichtig hatte schon Isabella Andorlini vielmehr an einzelne und nachträglich zusammengebundene Pergamentblätter in der Art einer Loseblattsammlung gedacht, nur daß die von ihr vermutete einseitige Beschriftung keineswegs zwingend erscheint. ${ }^{53}$ In jedem Fall mußte dies zugleich Einfluß auf die Heftung haben, da der Faden nicht gut durch den - hier eben nicht vorhandenen - Falz zu ziehen war; wie bei den aus Holztafeln gebildeten Polyptycha waren dazu vielmehr eigene Löcher durch den Freirand zu stechen. Ein instruktives Beispiel hierfür liegt in dem ledernen Notizbuch aus der Berliner Sammlung vor, dessen Abbildung Roberts und Skeat ihrer Behandlung des Phänomens beigegeben hatten. ${ }^{54}$ In der Spätantike begegnet dieses Format Jean Gascou zufolge auch bei Papyruscodices dokumentarischer Natur. ${ }^{55}$ Einzelblätter, keine Bifolia und nicht zuletzt das charakteristische Löcherpaar sind nun auch bei Frg. 2 und 3 des kürzlich edierten Pergamentcodex aus Antinoupolis anzutreffen, während das mit etwas breiterem Rand ausgestattete Frg. 1 allem Anschein nach einem anderen, dann sogar vierten Pergamentcodex angehörte. Damit wäre hierin der erste Beleg für ein Re-

${ }^{50}$ So bes. nach Galen, Comp.med.loc. I 1 (XII 423, 13-15 Kühn).

${ }^{51}$ Galen, Ind. 31-37 (11, 7-13, 2 Boudon-Millot/Jouanna).

${ }^{52} \mathrm{Zu}$ sehr an modernen Vorstellungen vom Buch orientiert Nicholls, Codices, bes. S. 384-5.

${ }^{53}$ So allerdings Andorlini, Precisazioni, bes. S. 413.

${ }^{54}$ Roberts/Skeat, Codex, 15 ff. sowie bes. S. 21 mit Anm. 2 und pl. II; eine Abb. auch bei Legras, Lire, 84 Fig. 43; die Ed. pr. des jetzt als SB XXVI 16551 (1. Hälfte III. Jhdt.; = TM\#97132) wiederabgedruckten Textes bei Poethke, Notizbuch, der die beiden $7,5 \mathrm{~cm}$ hohen und $6 \mathrm{~cm}$ breiten Lederstücke allerdings kaum zu recht als ,im Falz auseinandergeschnitten( )“ (so 399 in der Einl.) bzw. wiederholt als „Doppelblatt“ beschreibt. Auch das von Roberts/Skeat, a.a.O. angeführte Iliasfragment P.Berol. 10569 ist möglicherweise eher auf Leder als auf Pergament geschrieben, vgl. nur die unter $<$ http://ww2.smb.museum/berlpap/index.php/record/?result=0\&Alle=10569> zugänglichen Scans. Während daraus nichts zur Frage der Heftung zu ersehen ist, bestehen die beiden anderen dort erwähnten Papyruscodices wie üblich aus Bifolia mit Mittelfalz.

${ }^{55}$ Vgl. bes. Gascou, Codices, 79 bzw. 359 mit Verweis auf P.Sorb. II 69 (618/19 oder 633/34; = TM\#20110) und SB VI 9583 (2. Hälfte VIII. Jhdt. mit BL XII 198; = TM\#41014). 
zeptbuch aus einzelnen $\delta ı \varphi \theta \varepsilon ́ p \alpha ı$ zu sehen, das anfangs vielleicht tatsächlich, wie es der Name nahelegt und das Berliner Notizbuch bestätigt, aus einzelnen dünnen Lederstuicken bestand. Denn im Unterschied zu dem empfindlicheren Pergament war Leder nahezu unverwüstlich und ohne weiteres abwaschbar, so daß man es jederzeit bei sich führen und gleich wieder für neue Notizen nutzen konnte, sobald man die vorigen in ein passenderes Format übertragen hatte. ${ }^{56}$

Anders als die wissenschaftlichen Traktate waren Rezepte und Rezeptsammlungen offenbar das einzige Genos medizinischen Schrifttums, das bis weit in das Mittelalter hinein weiterlebte, wenngleich nunmehr in koptischer Sprache. ${ }^{57}$ Bemerkenswerterweise begegnet auch hier wieder die schon von den griechischen Papyri vertraute Zweitverwendung von Verwaltungstexten, so etwa bei einer "Handschrift in Buchform, für die arabische Urkunden mit leeren Rückseiten als Papier benutzt worden sind", wodurch wir ausnahmsweise genauere Kenntnis zu Zeit und Ort der Entstehung erlangen. ${ }^{58}$

Eine grundlegende Neuerung stellen indessen die gegen Ende des Jahrtausends auftauchenden Längsrollen dar. Bei der Edition eines $90 \mathrm{~cm}$ langen und $9 \mathrm{~cm}$ breiten Papyrus mit 27 Rezepten vermochte Tonio Sebastian Richter drei weitere, hier sogar annähernd drei Mal so breite Rotuli medizinischen oder alchemistischen Inhalts anzuführen, bei denen die Beschriftung ebenfalls meist auf der Rückseite weiterlief, die teilweise aber auch noch andere Texte - bei den alchemistischen Rollen arabische Briefe - auf dem Verso tragen. ${ }^{59}$ Hinsichtlich des Formats hatte Richter zugleich die Verwandtschaft zu den 200 Jahre früheren koptischen Rechtsurkunden vermerkt, sich jedoch mit dem Hinweis auf einen möglichen gemeinsamen Fundort im oberägyptischen Lepidotonpolis $45 \mathrm{~km}$ südlich von Sohag zufriedengegeben. ${ }^{60}$ Dasselbe Format begegnet andererseits auch im magischen Bereich. Man denke nur an den großen koptischarabischen Papyrus aus der Heidelberger Sammlung, der auf beiden Seiten insgesamt neun „Rezepte“ für Liebesund Vernichtungszauber bietet, ${ }^{61}$ oder an den Pergamentcodex des Montserrat mit dem 2. Buch Samuel, der zu unbekanntem Zeitpunkt in einen Rotulus mit magi-

${ }^{56} \mathrm{Zu}$ Leder als Beschreibstoff jetzt bes. Jördens/Kiyanrad/Quack, Leder.

57 So jetzt ausdrücklich Richter, Approach, bes. S. 38: „A shared feature (sc. mit der gleichzeitigen griechischen Evidenz) is the particular genre of medical writing in Coptic: our corpus consists exclusively of prescriptions - recipes for drugs, potions, plasters, and ointments."

${ }^{58}$ Vgl. A. Erman, Einl. zu BKU I 26, wobei sich die zeitliche Einordnung aus der Erwähnung des von 996 bis 1021 amtierenden Kalifen al-Hakim, der Ort aus dem gemeinsamen Ankauf mit weiteren Texten aus dem Faijumort Tutun und dem für dieses Gebiet typischen Dialekt ergibt.

59 Richter, Texte, 167 mit Verweis auf die sämtlich dem IX./X. Jhdt. zugewiesenen P.Méd.Copt.IFAO (248 x 27 cm, vgl. auch ebda. S. 190 Nr. 22; = TM\#108434) und die beiden noch in Bearbeitung befindlichen alchemistischen Rotuli Bodl. Ms. Copt. a.2 (P) $(81$ x $25 \mathrm{~cm}$; = TM\#112407) und a.3 (P) (71 x 25 cm; = TM\#112408), zu den Maßen bes. Anm. 39; der Hinweis auf den jeweils auf der Rückseite niedergelegten „Arabic letter“ P.Bodl.Arab. 1 bzw. 2 in $<$ www.trismegistos.org $>$ unter den TM-Nummern.

${ }^{60}$ Richter, Texte, 167-8.

${ }^{61}$ VBP [P.Bad.] V 123 (VIII. Jhdt.?, 121 x 21 cm; = TM\#102087); hierzu zuletzt Mößner/Nauerth, Texte, bes. S. $306,314 \mathrm{ff}$. 
schen Formeln und Zeichnungen umgearbeitet wurde. ${ }^{62}$

Sehr viel besser sind wir über dieses Format jetzt durch die Forschungen zur Kairener Genizah orientiert, wo gerade die Rotuli, ob aus Pergament oder aus Papier, in den letzten Jahren verstärkte Aufmerksamkeit erfuhren. ${ }^{63}$ Inzwischen ließen sich Hunderte zugehöriger Fragmente mit typischen Gebrauchstexten identifizieren. Mehr als die Hälfte enthält demnach liturgische Dichtungen oder Zeugnisse persönlicher Frömmigkeit, daneben finden sich Kommentare, lexikographische Werke, Rechtsauskünfte, aber eben auch wieder magische, astrologische und medizinische Texte. Erneut treffen wir auf wiederverwendete Verwaltungsrollen, beidseitige Beschriftung und wenig stilbewußte Hände, wie denn auch eher praktische als ästhetische Zwecke im Vordergrund standen, ja sogar Leder- und Pergamentstücke gemeinsam zu einer Rolle verarbeitet wurden. All dies deutet auf hohen Bedarf bei intensiver Verwendung und hohem Verschleiß, woraus schon Judith Olszowy-Schlanger ein Bild des typischen Nutzers entwarf: „The cheap rotulus book form appears to have been favoured by the highly literate, the intellectual elites, for their personal devotion, study and work." ${ }^{\text {64 }}$ Leicht und transportabel, kostengünstig und vor allem jederzeit durch zusätzliche Blätter erweiterbar, sind in diesen Rotuli letztlich die Nachfolger von Galens $\pi$

Nach alldem stellt sich das Spektrum der Schriftträger und Buchtypen im medizinischen Bereich noch einmal vielfältiger dar, als die übliche Dreiheit von Einzelblatt, Rolle und Codex es vermuten ließe. Schon die wenigen hier unternommenen Stichproben haben gezeigt, wie viele Einblicke gerade ein solches Schrifttum erlaubt, das auf der Schwelle zwischen literarischer und dokumentarischer Evidenz steht, und welche Anknüpfungspunkte und neue Perspektiven dies wiederum bietet; wie sich zudem die Arten des Zugriffs und demnach offenbar auch die Bedürfnisse veränderten, die die Gesellschaft an das medizinische Wissen stellte. So zeichnet sich einerseits eine gewisse Tendenz zur 'Kanonisierung' der Schriften der großen Ärzte ab, andererseits aber auch eine zunehmende Ablösung anspruchsvollerer wissenschaftlicher Spezialliteratur durch relativ einfach handhabbare Rezepte und Rezeptsammlungen, die in Richtung einer Haus- oder auch Reiseapotheke gehen. ${ }^{65}$

Krankheiten machen bekanntlich vor niemandem halt, und zu allen Zeiten und

${ }^{62}$ TM\#126174 mit P.Monts.Roca II 3+4+6+7, derzeit in Bearbeitung durch Korshi Dosoo; vgl. auch Jones, Fragment.

${ }^{63}$ Hierzu wie zum folgenden Bohak, Rotuli, und bes. Olszowy-Schlanger, Form sowie zuletzt dies., Books; vgl. auch dies., Rotulus. Herzlicher Dank geht an Anna Busa, die mich nicht nur erstmals auf dieses Material aufmerksam machte, sondern auch mit Literatur unterstützte.

${ }^{64}$ Olszowy-Schlanger, Books, bes. S. 100.

${ }^{65} \mathrm{Vgl}$. auch schon Marganne, Livre médical, $127 \mathrm{ff}$. im „Épilogue“ und bes. zusammenfassend 130 f., wiewohl mit Blick allein auf die Evidenz in den klassischen Sprachen; zum Nutzen auf Reisen bes. das Proömium der wohl im späteren III. Jhdt. entstandenen, unter dem Namen des Plinius Secundus Iunior überlieferten und immer wieder ergänzten Medicina Plinii, jetzt bequem zugänglich in der zweisprachigen Ausgabe von Brodersen, Reiseapotheke. 
in allen Sprachen und Gesellschaften bemühte man sich, ihnen zu begegnen und das einmal erlangte Wissen darüber auf Dauer zu fixieren. Das hier in den Blick genommene Material läßt freilich zugleich erkennen, daß jede Epoche ihre jeweils eigenen Formen dafür entwickelte und der Umgang mit diesem Wissen im Laufe der Jahrhunderte stetem Wandel unterworfen war. So bleibt auch nach dem erfolgreichen Abschluß des DIGMEDTEXT-Projekts noch genug zu tun, was auf diesem Gebiet der Erforschung harrt, und es steht zu hoffen, daß dies auch weiterhin die gebührende Anerkennung und alle dazu notwendige Unterstützung findet.

\section{Literatur}

Andorlini, Precisazioni = Isabella Andorlini, Precisazioni sulla data di alcuni testi di medicina in forma di codice, in: A. Bülow-Jacobsen (Hrsg.), Proceedings of the XX Internationa Congress of Papyrology (Copenhagen 23.-29. 8. 1992), Copenhagen 1994, 410-13.

Andorlini Marcone, Apporto = Isabella Andorlini, L'apporto dei papiri alla conoscenza della scienza medica antica, in: W. Haase (ed.), Aufstieg und Niedergang der römischen Welt, II 37.1, Berlin/New York 1993, 458-562.

Beltz, Katalog $=$ Walter Beltz, Katalog der koptischen Handschriften der Papyrus-Sammlung der Staatlichen Museen zu Berlin (Teil I), APF 26 (1978), 57-119; (Teil II), APF 27 (1980), 121-222.

Bohak, Rotuli = Gideon Bohak, The Magical Rotuli from the Cairo Genizah, in: G. Bohak / Y. Hariri / Sh. Shaked (Hrsg.), Continuity and Innovation in the Magical Tradition (Jerusalem Studies in Religion and Culture 15), Leiden/Boston 2011, 321-40.

Brodersen, Reiseapotheke = Kai Brodersen, Plinius' kleine Reiseapotheke, Stuttgart 2015.

Cavallo, Calamo = Guglielmo Cavallo, Il calamo e il papiro. La scrittura greca dall'età ellenistica ai primi secoli di Bisanzio (Pap. Flor. 36), Firenze 2005.

Del Corso/Pintaudi, Papiri = Lucio Del Corso / Rosario Pintaudi, Papiri letterari dal Museo Egizio del Cairo e una copertina di codice da Antinoupolis, in: L. Del Corso / F. De Vivo / A. Stramaglia (Hrsg.), Nel segno del testo. Edizioni, materiali e studi per Oronzo Pecere (Pap. Flor. 44), Firenze 2015, 3-29.

Dorandi, Tradierung = Tiziano Dorandi, Tradierung der Texte im Altertum; Buchwesen, in: H.-G. Nesselrath (Hrsg.), Einleitung in die griechische Philologie, Stuttgart/Leipzig 1997, 3-16.

Fournet, Antinoupolis = Jean-Luc Fournet, I papiri di Antinoupolis. La collezione e gli scavi fiorenti$n i$, in: G. Bastianini / A. Casanova (Hrsg.), 100 anni di istituzioni fiorentine per la papirologia. Atti del convegno internazionale (Firenze, 12.-13. 06. 2008) (STP N.S. 11), Firenze 2009, 115-32.

Fournet, Lambeaux = Jean-Luc Fournet, Ces lambeaux, gardiens de la mémoire des hommes. Papyrus et culture de l'Antiquité tardive, Paris 2016.

Gascou, Codices = Jean Gascou, Les codices documentaires égyptiens, in: A. Blanchard (Hrsg.), Les débuts du codex (Bibliologia 9), Turnhout 1989, 71-101 = ders., Fiscalité et société en Égypte byzantine, Paris 2008, 351-76.

Hagen, Ostraca $=$ Fredrik Hagen, New Kingdom Ostraca from the Fitzwilliam Museum, Cambridge, Leiden/Boston 2011.

Jördens, Texte = Andrea Jördens, Griechische Texte aus Ägypten, in B. Janowski / D. Schwemer (Hrsg.), Texte aus der Umwelt des Alten Testaments, Neue Folge Band 5. Texte zur Heilkunde (TUAT.NF 5), Gütersloh 2010, 317-50.

Jördens/Kiyanrad/Quack, Leder = Andrea Jördens / Sarah Kiyanrad / Joachim F. Quack, Leder, in: Th. Meier / M. R. Ott / R. Sauer (Hrsg.), Materiale Textkulturen. Konzepte - Materialien Praktiken (Materiale Textkulturen 1), Berlin/München/Boston 2015, 323-35. 
Jonckheere, Prescriptions $=$ Frans Jonckheere, Prescriptions médicales sur ostraca hiératiques, CE 29 (1954), 46-61.

Jones, Fragment = Brice C. Jones, A New Coptic Fragment of 2 Samuel 10:13-14, 17-18: McGill MS NO Coptic 2, ZPE 184 (2013), 126-30.

Legras, Lire = Bernard Legras, Lire en Égypte, d'Alexandre à l'Islam, Paris 2002.

Leith, Herbal $=$ David Leith, The Antinoopolis Illustrated Herbal (PJohnson + PAntin. 3. $214=$ $M P^{3}$ 2095), ZPE 156 (2006), 141-56.

Marganne, Collection = Marie-Hélène Marganne, La 'collection médicale' d'Antinoopolis, ZPE 56 (1984), 117-21.

Marganne, Hippocrate = Marie-Hélène Marganne, Hippocrate dans un monde de chrétiens: la réception des traités hippocratiques dans la chôra égyptienne à la période byzantine (284-641), in: J. Jouanna / M. Zink (Hrsg.), Hippocrate et les hippocratismes: médecine, religion, société. Actes Coll. AIBL (Paris 8.-10. 11. 2012), Paris 2014, 283-307.

Marganne, Livre médical = Marie-Hélène Marganne, Le livre médical dans le monde grécoromain (Cahiers du CeDoPal 3), Liège 2004.

Menci, Antinoe = Giovanna Menci, I papiri letterari 'sacri' e 'profani' di Antinoe, in: L. Del Francia Barocas (Hrsg.), Antinoe cent'anni dopo. Catalogo della mostra (Firenze, 10. 07. - 01. 11. 1998), Firenze 1998, 49-55.

Mößner/Nauerth, Texte = Tamara Mößner / Claudia Nauerth, Koptische Texte und ihre Bilder, in: A. Jördens (Hrsg.), Ägyptische Magie und ihre Umwelt (Philippika 80), Wiesbaden 2015, 302-75.

Morelli, P. Ant. III 139 = Federico Morelli, P. Ant. III 139 fr. 1: un frammento di Galeno, De compositione medicamentorum per genera VII 10 - e di P. Ant. III 186?, ZPE 172 (2010), 203-7.

Nicholls, Codices $=$ Matthew Nicholls, Parchment Codices in a New Text of Galen, G\&R 57 (2010), 378-86.

Olszowy-Schlanger, Books = Judith Olszowy-Schlanger, Cheap Books in Medieval Egypt: Rotuli from the Cairo Geniza, "Intellectual History of the Islamicate World" 4 (2016), 82-101.

Olszowy-Schlanger, Form = Judith Olszowy-Schlanger, The Third Form of the Hebrew Book: rotuli from the Cairo Genizah, in: Report of the Oxford Centre for Hebrew and Jewish Studies, Academic Year 2010-2011, Oxford 2011, 89-93.

Olszowy-Schlanger, Rotulus = Judith Olszowy-Schlanger, Un rotulus du midrash Pirqa deRabbenu ha-Qadosh de la Geniza du Caire, "Annuaire de l'École pratique des hautes études, Section des sciences historiques et philologiques" 145 (2014), 26-40.

Poethke, Notizbuch = Günter Poethke, Ein Berliner ,Notizbuch' aus Leder, in: C.-B. Arnst / I. Hafemann / A. Lohwasser (Hrsg.), Begegnungen. Antike Kulturen im Niltal (Festgabe E. Endesfelder - K.-H. Priese - W. F. Reineke - S. Wenig), Leipzig 2001, 399-403.

Préaux, Préscriptions = Claire Préaux, Les préscriptions médicales des ostraca grecs de la Bibliothèque Bodléenne, CE 31 (1956), 135-48.

Quack, Kontinuität = Joachim F. Quack, Kontinuität und Wandel in der spätägyptischen Magie, "Studi epigrafici e linguistici sul Vicino Oriente antico" 15 (1998), 77-94.

Quibell, Excavations = James E. Quibell, Excavations at Saqqara (1997-1908), Le Caire 1909.

Richter, Approach = Tonio S. Richter, Toward a Sociohistorical Approach to the Corpus of Coptic Medical Texts, in: M. Ayad (Hrsg.), Studies in Coptic Culture: Transmission and Interaction, Cairo 2016, 33-54.

Richter, Texte = Tonio S. Richter, Neue koptische medizinische Texte, ZÄS 141 (2014), 154-94.

Ritner, Mechanics = Robert K. Ritner, The Mechanics of Ancient Egyptian Magical Practice (SAOC 54), Chicago 1993.

Roberts/Skeat, Codex = Colin H. Roberts / Theodore C. Skeat, The Birth of the Codex, London 1983.

Turner, Typology = Eric G. Turner, The Typology of the Early Codex, Philadelphia 1977.

Westendorf, Handbuch = Wolfhart Westendorf, Handbuch der altägyptischen Medizin (HdO 1.36), 2 Bde., Leiden/Boston/Köln 1999. 